\title{
Pheochromocytoma, Paraganglioma, Neuroblastoma, Catecholamine Secreting Tumor Perioperative and Anesthetic Management in Children
}

\section{Claudine Kumba (iD)}

Department of Pediatric Anesthesia and Critical Care, Necker Enfants Malades University Hospital, Paris, France

\section{Abstract}

Background: Catecholamine secreting tumors are responsible of $0.5-2 \%$ of hypertension in children. Intraoperative instability in these patients during surgical resection can occur. The objective of this mini-review was to describe the perioperative management of pheochromocytomas, paragangliomas, neuroblastomas and catecholamine secreting tumors in children.

Methods: Narrative mini-review.

Results and Conclusion: Preoperative medical and intraoperative anesthetic management aim to reduce intraoperative instability in catecholamine secreting tumors.

\section{Publication History:}

Received: July 09, 2019

Accepted: August 19, 2019

Published: August 21, 2019

\section{Keywords:}

Pheochromocytomas, Paraganglioma, Neuroblastoma, pediatric anesthesia, Crtical care

\section{Introduction}

In children, pheochromocytomas (PH) constitute $80-85 \%$ of catecholamine secreting tumor (CST) originating from the chromaffin cells of the adrenal gland medulla; whereas paragangliomas (PG) constitute $5-10 \%$ of the extra adrenal pheochromocytomas and are originated from the sympathetic (in the chest, abdomen and pelvis) and parasympathetic ganglia (in the head and neck) and are rarely functional and secreting; neuroblastoma (NB) is a neuroendocrine tumor from the adrenal gland or primitive neural crest elements of the sympathetic nervous system [1-3]. These CST are responsible of $0.5-2 \%$ of the hypertension in the pediatric population. These tumors if they are functional secrete dopamine, norepinephrine and epinephrine [3]. The average age of presentation is 11-13 years, with boys being concerned twice than girls. Symptoms and signs are due to the circulating catecholamines and present as hypertension (sustained rather than paroxysmal), tachycardia, cardiac conduction anomalies, headaches, sweating, flushing, nausea, vomiting, dizziness , anxiety, weight loss, visual disturbance, polyuria, polydipsia, dysphagia due to mass effect, hypertrophic and dilated cardiomyopathy, arrhythmias and renal impairement [2-4].

Diagnostic tests realized for screening consist of free plasma and 24 hours urinary catecholamine metabolites dosage namely metanephrine and normetanephrine. Measurements of plasma and $24 \mathrm{~h}$ urinary catecholamines and and urinary vanillylmandelicacid are no longer recommended $[3,4]$.

Imaging with CT scan and or magnetic resonance imaging (MRI) contribute to the localization and identification lesions $[2,3]$.

Genetic screening is recommended for two reasons [2,3]. Firstly, $80 \%$ of PH and PG are inherited namely multiple endocrine neoplasia (MEN) type 2, Von Hippel-Lindau (VHL) type 2, neurofibromatosis (NF) type1, and paraganglioma-pheochromocytoma syndromes (SDHx). Secondly, malignancy rates can reach $12-47 \%$.

Benign tumors have 5-15 years survival rate of $100 \%$ whereas malignant tumors (metastases, unresectable tumors, recurrent tumors after resection) have 5,10 , and 15 year survival rate of 78,62 , and $31 \%$ respectively.

Treatment in these lesions depends on the characteristic of the tumor. Surgery with complete resection is curative [3]. Radiotherapy with radionuclide therapy or external beam radiotherapy and chemotherapy with cyclophosphamide, vincristin and dacarbazine are reserved for malignant tumors [3].

Surgical treatment for these tumors is organized on an elective setting to optimize the patient's status especially in the CST and malignant tumors.

\section{Description of the Perioperative Management}

Prior anesthetic consultation several weeks (at least 1 week before surgery for optimal management) is mandatory with complementary laboratory tests namely hemoglobin and platelet levels, blood urea nitrogen to assess for renal impairment, ions, glycemia, cross match (intraoperative hemorrhage is possible), electrocardiogram and echocardiography (to assess for arrhythmias and cardiomyopathy due to chronic exposure to catecholamines) [1-4].

In case of a functional $\mathrm{PH} / \mathrm{PG}$ (CST), appropriate preoperative medical management reduces perioperative complications (severe hypertensive crisis, hypotension, arrhythmias, cerebrovascular accidents) from $69 \%$ to $3 \%$ with the use of a blockade $[2,4]$. Patients should be admitted to hospital 24-36h prior to surgery and given an alpha blocker, beta blocker, (and in some cases a tyrosine hydroxylase inhibitor) the night before surgery if the tumor is secreting. Monitoring hemodynamic and fluid therapy to prevent hypotension can be necessary [4]. The goal of the medical treatment is to reduce the symptoms and signs related to catecholamine secretion in functional tumors. The preoperative medical treatment can consist of three phases according to the severity of the symptoms and signs.

"Corresponding Author: Dr. Claudine Kumba, Department of Pediatric Anesthesia and Critical Care, Necker Enfants Malades University Hospital, Paris, France; E-mail: claudine.kumba@gmail.com

Citation: Kumba C (2019) Pheochromocytoma, Paraganglioma, Neuroblastoma, Catecholamine Secreting Tumor Perioperative and Anesthetic Management in Children. Int J Pediatr Neonat Care 5: 156. doi: https://doi.org/10.15344/24552364/2019/156

Copyright: (c) 2019 Kumba. This is an open-access article distributed under the terms of the Creative Commons Attribution License, which permits unrestricted use, distribution, and reproduction in any medium, provided the original author and source are credited. 
Citation: Kumba C (2019) Pheochromocytoma, Paraganglioma, Neuroblastoma, Catecholamine Secreting Tumor Perioperative and Anesthetic Management in Children. Int J Pediatr Neonat Care 5: 156. doi: https://doi.org/10.15344/2455-2364/2019/156

Page 2 of 2

Initially phenoxybenzamine, anon competive $\alpha 1$ and $\alpha 2$ blocker can be administered with a starting dose of $0.2 \mathrm{mg} / \mathrm{kg} /$ day (maximum $10 \mathrm{mg} /$ dose) orally with a maintenance dose increased by $0.2 \mathrm{mg} /$ $\mathrm{kg} /$ day every 4 days to reach $0.4-1.2 \mathrm{mg} / \mathrm{kg} /$ day every $6-8$ hours (maximum $2-4 \mathrm{mg} / \mathrm{kg} /$ day) orally. Common side effects are orthostatic hypotension, tachycardia and nasal congestion. An alternative to phenoxybenzamine is doxazosin a selective alpha-1 blocker with a starting dose of $1-2 \mathrm{mg} /$ day orally with a maintenance dose increased to $4 \mathrm{mg}$ daily or in 2 times daily orally. Common side effects of this drug are orthostatic hypotension and dizziness.

If alpha blockers do not control the hypertension, a calcium blocker can be added in a second phase. Monotherapy with calcium channel blockers is not recommended unless patients have very mild preoperative hypertension or have severe orthostatic hypotension with adrenergic receptor blockers. Amlodipine can be the drug of choice with a starting dose of $5 \mathrm{mg}$ /day orally and maximum dose of $10 \mathrm{mg} /$ day orally.

In the third phase beta blockers can be added at least 3 days prior to surgery to control and prevent arrhythmias and reflex tachycardia due to a blockade. The selective beta-1 blocker atenolol can be the drug of choice with a starting dose of $0.5-1 \mathrm{mg} / \mathrm{kg} / \mathrm{day}$, daily or in 2 times daily orally with a maintenance dose of $2 \mathrm{mg} / \mathrm{kg} /$ day, up to $100 \mathrm{mg} /$ day, daily or in 2 times daily orally. Common side effects of this drug are edema, dizziness and fatigue. An alternative to atenolol is the non-selective beta blocker propranolol with a starting dose of $1-2 \mathrm{mg} / \mathrm{kg} /$ day, in $2-4$ times daily orally with a maintenance dose of $4 \mathrm{mg} / \mathrm{kg} / \mathrm{day}$, up to $640 \mathrm{mg} /$ day, in $2-4$ times daily orally. Common side effects are dizziness, fatigue and asthma exacerbation. Once the hypertension is controlled surgery is scheduled electively. The latter can be laparoscopy or laparotomy according to the size of the tumor [4].

\section{Anesthetic Management}

The goals of the intraoperative management are to avoid hypertensive crisis and tachycardia during induction of anesthesia and tumor resection and manipulation. Esmolol or nitroprusside (or magnesium sulfate or dexmedetomidine or nicardipine) can be used intravenously [1-4]. Hypotension can be avoided with goal directed fluid therapy (with crystalloids) and vasopressors: fluid therapy in case of catecholamine induced dilated cardiomyopathy should be used cautiously and monitoring goal directed fluid therapy with oesophageal doppler probe can be mandatory [5]. Hyperglycemia is common in CST and monitoring this parameter is important.

Cross match, PRBC (packed red blood cells) must be available on the day of surgery because intraoperative hemorrhage is possible.

Monitoring with electrocardiogram, pulse oximetry (SPO2), invasive arterial blood pressure, central venous catheter ( for vasopressors administration), two large bore peripheral intravenous accesses, oesophageal doppler [5] probe (to monitor goal directed fluid therapy), temperature, arterial gas monitoring. Epidural or paravertebral catheter (in case of laparotomy but can be also interesting intraoperatively in case of laparoscopy to avoid opioids). Hypotension is less likely with paravertebral block.

Induction of anesthesia: IV access prior to induction must be available: IV or inhalational (in a smooth manner), maintenance with Inhalational, opioids (sufentanil, remifentanil), muscle relaxants(cisatracurium, rocuronium during surgery) and epidural or paravertebral catheter for intraoperative and postoperative analgesia management.

Some drugs can precipitate crisis in pheochromocytoma (4) namely:

1. Dopamine D2 receptor antagonists: metoclopramide, sulpiride, amisulpride, tiapride, chlorpromazine, prochlorperazine, droperidol

2. Adrenergic receptor blockers, sympathomimetics: propranolol (prior to a blockade), sotalol, timolol, nadolol, labetalol, ephedrine, pseudoephedrine, fenfluramine, methylphenidate, phentermine, dexamfetamine.

3. Opioid analgesics: morphine, pethidine, tramadol

4. Norepinephrine reuptake inhibitors (including tricyclic antidepressants): amitriptyline, imipramine

5. Serotonin reuptake inhibitors (rarely reported): paroxetine, fluoxetine

6. Monoamine oxidase inhibitors: tranylcypromine, moclobemide, phenelzine

7. Corticosteroids: dexamethasone, prednisone, hydrocortisone, betamethasone

8. Peptides: ACTH, glucagon

9. Neuromuscular blocking agents: succinylcholine, tubocurarine, atracurium

Postoperative management aim to avoid:

1. Hypotension due to preoperative a blockade with goal directed fluid therapy and or vasopressors.

2. Hypoglycemia due to rebound hyperinsulinism related to reduction in circulating catecholamines.

\section{Conclusion}

Perioperative management of pheochromocytomas, paragangliomas, neuroblastomas and catecholamine secreting tumors depends on the characteristic of the lesion. In case of CST preoperative hemodynamic status optimization with alpha, calcium and beta blockers can be necessary to avoid intraoperative instability. Intraoperative fluid and hemodynamic goal directed therapy is important because of the possible cardiovascular impact of CST during tumor resection.

\section{Competing Interests}

The author declare no competing interests.

\section{References}

1. Hack HA (2000) The perioperative management of children with phaeochromocytoma. Paediatr Anaesth 10: 463-476.

2. Romero M, Kapur G, Baracco R, Valentini RP, Mattoo TK, et al. (2015) Treatment of Hypertension inChildren With Catecholamine-Secreting Tumors: A Systematic Approach. J Clin Hypertens 17: 720-725.

3. Bholah R, Bunchman TE (2017) Review of Pediatric Pheochromocytoma and Paraganglioma. Front Pediatr 5: 155.

4. Lenders JWM, Duh OY, Eisenhofer G, Gimenez-Roqueplo AP, Grebe SKG, et al. (2014) Pheochromocytoma and Paraganglioma: An Endocrine Society Clinical Practice Guideline. J Clin Endocrinol Metab 99: 1915-1942.

5. Hack H (2006) Use of the Esophageal Doppler Machine to help guide the intraoperative management of two children with pheochromocytoma. Pediatric Anesthesia 16: 867-876. 